Research Article

\title{
Development and Performance Analysis of Pneumatic Soft-Bodied Bionic Actuator
}

\author{
Wenchuan Zhao $\left(\mathbb{D}\right.$, Yu Zhang $\mathbb{D}^{\mathbb{D}}$, and Ning Wang \\ School of Mechanical Engineering, Shenyang University of Technology, Shenyang 110870, China \\ Correspondence should be addressed to Yu Zhang; zhangyu@sut.edu.cn
}

Received 10 December 2020; Revised 22 January 2021; Accepted 9 February 2021; Published 19 February 2021

Academic Editor: Donato Romano

Copyright (c) 2021 Wenchuan Zhao et al. This is an open access article distributed under the Creative Commons Attribution License, which permits unrestricted use, distribution, and reproduction in any medium, provided the original work is properly cited.

\begin{abstract}
The design of a pneumatic soft-bodied bionic actuator derives from the structural characteristics and motion mechanism of biological muscles, combined with the nonlinear hyperelasticity of silica gel, which can improve the mobility and environmental adaptability of soft-bodied bionic robots. Based on Yeoh's second-order constitutive model of silica gel, the deformation analysis model of the actuator is established, and the rationality of the structure design and motion forms of the actuator and the accuracy of the deformation analysis model are verified by using the numerical simulation algorithm. According to the physical model of the pneumatic soft-bodied bionic actuator, the motion and dynamic characteristics of the actuator are tested and analyzed, the curves of motion and dynamic characteristics of the actuator are obtained, and the empirical formula of the bending angle and driving torque of the actuator is fitted out. The results show that the deformation analysis model and numerical simulation method are accurate, and the pneumatic soft-bodied bionic actuator is feasible and effective, which can provide a design method and reference basis for the research and implementation of soft-bodied bionic robot actuator.
\end{abstract}

\section{Introduction}

The traditional robot is composed of a large number of rigid parts, which face the disadvantages of low human-computer interaction security, poor adaptability in unstructured environment, low driving efficiency, and high maintenance cost and has gradually failed to meet the actual needs. With the thriving of bionic robot technology, material technology, and rapid prototyping technology, the soft-bodied bionic robot with high flexibility, safety, and efficiency has become a new direction in the field of robot development [1-4]. Among them, the actuator is the main component in realizing the motion function of the soft-bodied bionic robot. Therefore, in order to improve the maneuverability of the soft-bodied bionic robot, researchers have developed different types of actuators $[5,6]$.

Najem and Leo [7] from the Virginia Institute of Technology have developed an IPMC actuator controlled by electrical signals. Through the bending deformation of the IPMC actuator, the soft-bodied bionic jellyfish robot can achieve contraction and expansion. Shepherd et al. [8] from the Har- vard University have developed an actuator driven by chemical explosion. Through the explosion impact of chemical fuel, the soft-bodied bionic robot can realize the jumping motion. Villanueva et al. [9] from the University of Virginia have developed an actuator driven by an electric motor, which enables the soft-bodied bionic robot to realize underwater propulsion and swimming posture adjustment. Renda et al. [10] from the University of Khalifa have developed an actuator driven by cables, through imitating the motion mechanism of biological arms; the soft-bodied bionic robot can realize amphibious mobility. Researchers [11] from BEAR Laboratory of the University of Maine have developed a voltage-controlled composite material actuator made from ionic polymers and nanometal. Through the bending deformation of the material body, the soft-bodied bionic robot can achieve the required motion forms. Wehner et al. [12] from the Weiss Institute of Bioengineering in Harvard University have developed an actuator based on platinum catalytic reaction, which enables the soft-bodied bionic octopus robot to realize position and orientation transformation. Rodrigue et al. [13] from the Seoul National University have 
developed an actuator driven by a memory alloy material. By inserting two memory alloy wires with opposite eccentric angles inside the polymer, the soft-bodied bionic robot is capable of performing related tasks. Wang et al. [14] from the Tsinghua University have developed an actuator driven by adding a certain amount of ethanol to the liquid metal composite materials. Based on the principle that ethanol volatilizes with the change of temperature, the soft-bodied bionic octopus robot can achieve the change of posture. Musta et al. [15] from the Tartu University have developed an actuator driven by the electrical excitation process of ion penetration, and the driving process is cyclic reversible. Yichuan et al. [16] from the Shenzhen University of Geneva Raklev have developed an actuator driven by the piezoelectric chip structure under the action of AC voltage. Based on the principle of piezoelectric contraction, the position and pose of the soft-bodied bionic robot can be adjusted.

The driving methods of the above soft-bodied bionic robot actuators can be classified as chemical driven, intelligent material driven, and electric driven. These driving methods are mainly faced with low driving efficiency, poor stability, and limited pose transformation. Therefore, pneumatic and hydraulic driven soft-bodied actuators and the corresponding soft-bodied bionic robots are gaining favor, because of their outstanding characteristics of simple driving methods, high driving efficiency, strong environmental adaptability, and smooth posture transformation.

The soft-bodied bionic underwater robot [17-19] driven by pneumatic and hydraulic actuators can fully imitate the structural characteristics and motion mechanism of underwater organisms. Through the study of its swing frequency and amplitude, the soft-bodied robot can achieve high driving efficiency and stability. The soft-bodied bionic crawling robot driven by pneumatic and hydraulic actuators [20-22] can fully imitate the structural characteristics and motion mechanism of worms. Through the study of the relationship between friction and driving force, the soft-bodied robot can realize relevant operations in planes, pipeline, and other environments. The snake-like soft-bodied bionic robot driven by pneumatic and hydraulic actuators $[23,24]$ can fully simulate the structural characteristics and motion mechanism of snake-like organisms. Through its zigzag form of pose transformation, the soft-bodied robot can work flexibly in narrow spaces. The wheel-walking soft-bodied bionic robot driven by pneumatic and hydraulic actuators [25], based on its wheel-walking structural characteristics, can achieve rapid maneuvering through periodic bending changes of the body structure. The pneumatic and hydraulic driven actuators of soft-bodied bionic joint [26] can boost its power output by adjusting the output of the driving torque. The soft-bodied bionic robotic arm driven by pneumatic and hydraulic actuators $[27,28]$ controls the internal pressure of the driving cavity structure through the pressure proportional valve, so that it can realize flexible operation and has the function of elongation. The soft-bodied bionic robotic manipulator driven by pneumatic and hydraulic actuators $[29,30]$ can guarantee the grasping and releasing of single or multiple target objects without doing any damage to them. Pneumatic and hydraulic driven soft-bodied bionic basic execution unit [31-33] can realize the basic driving modes and purposes according to the driving cavity of different types of designed structure.

Although the abovementioned pneumatic and hydraulic driven soft-bodied bionic actuators can meet the corresponding design requirements, there exist drawbacks including poor environmental adaptability, single motion form, and lack of systematic research methods. Besides, most of the researches are limited to the type of entirely soft-bodied or filament+soft-bodied, which calls for the improvement of structure strength, execution strength, and resilience of the pneumatic soft-bodied bionic actuators, making it difficult to lay a good foundation for further application. Therefore, this paper deriving from the nature of structural characteristics and motion mechanism of biological muscles, and making full use of the advantages of the silica gel material, the structure design, mechanical model, numerical simulation algorithm verification, physical model preparation, experimental test analysis of the pneumatic soft-bodied bionic actuator are carried out while simplifying the structure of the bionic body, so as to greatly improve its environmental adaptability and motion performance and broaden its application field. Besides, the pneumatic network devices of the actuator are independent of each other, which can not only effectively avoid mutual coupling interference but also improve the motion performance. Therefore, this paper can provide a reference for the research of pneumatic softbodied bionic robot actuator, especially through the theoretical modeling of the soft-bodied actuator embedded with pressure spring structure; it can also provide a new idea for the theoretical research of the soft-bodied actuator. It should be noted that the actuator can be used in mobile robots and manipulating robots. For the mobile robot equipped with the actuator, in the underwater environment, it can simulate the motion form of the torso undulating type fishtail and the torso swinging type fishtail, so that the robots can swim and turn the rudder quickly; in the land environment, it can simulate the motion mechanism of the inchworm, so that the robots can crawl and change direction. For the manipulative robots equipped with this actuator, different numerical pressure can be applied to the middle, left, and right actuator units, so that the manipulator can complete certain work requirements.

\section{Structure Design and Motion Forms Analysis}

2.1. Structure Design. The pneumatic soft-bodied bionic actuator is shown in Figure 1. Its structure is mainly composed of three pneumatic network devices, a nonretractable column structure, and a compression spring structure. Each actuating unit is composed of a pneumatic network device, a nonretractable column structure, and a compression spring structure, and each pneumatic network device is composed of 13 driving cavity structures. Besides, in order to enhance the structural strength, execution force, and motion restoring force of the actuator, the compression spring structure is embedded in the inner part of the nonretractable column.

The main structural parameters of the actuator are shown in Table 1. It should be noted that in order to ensure the 


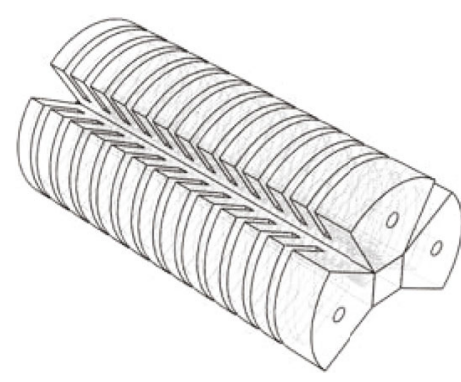

(a)

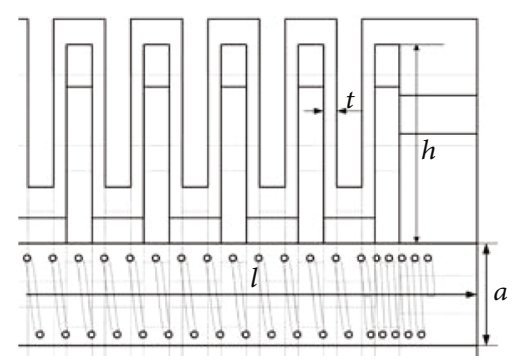

(b)

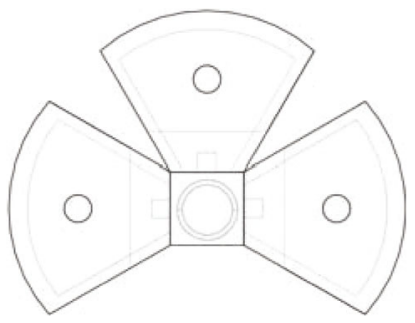

(c)

FIGURE 1: Structure diagram of pneumatic soft-bodied bionic actuator. (a) Overall structure of actuator. (b) Partial view of actuator cross section. (c) End view of actuator.

TABLE 1: Main structural parameters of actuator.

\begin{tabular}{lcc}
\hline No. & Size & Numerical values \\
\hline 1 & Driving cavity structure internal height $h$ & $35 \mathrm{~mm}$ \\
2 & Driving cavity structure expansion wall thicknesst & $2 \mathrm{~mm}$ \\
3 & Driving cavity structure expansion wall bottom height $h_{a}$ & $8 \mathrm{~mm}$ \\
4 & Nonretractable column structure thickness $a$ & $16 \mathrm{~mm}$ \\
5 & Nonretractable column structure length $l$ & $160 \mathrm{~mm}$ \\
6 & Driving cavity structure number $N$ & 13 \\
\hline
\end{tabular}

interaction between the driving cavity structures of actuator, the other walls of the driving cavity structures are required to be thicker than the expansion walls.

2.2. Analysis of Motion Forms. The motion process of the pneumatic soft-bodied bionic actuator can be divided into two parts: the middle part periodic bending and the left and right sides periodic bending. In the middle part periodic bending motion stage, when the air pump inflates the inner part of the middle part actuating unit of the actuator, driving cavity structures to interact with each other, resulting in the expansion and bending deformation of the middle part of the actuator, as shown in Figure 2(a). In the left side periodic bending motion stage, when the air pump inflates the left side actuating unit of the actuator, the left side of the actuator will expand and bend due to the interaction of the driving cavity structures, as shown in Figure 2(b). In the right side periodic bending motion stage, when the air pump inflates into the right side actuating unit of the actuator, the right side of the actuator will expand and bend due to the interaction of the driving cavity structures, as shown in Figure 2(c).

\section{Mechanical Model Analysis}

3.1. Constitutive Model of Silica Gel Material. The pneumatic soft-bodied bionic actuator is mainly composed of silica gel material, which has the characteristics of hyperelasticity and the capability of large deformation. There exists a highly nonlinear relationship between its strain and stress. In order to ensure the reliability of the research results, the phenomenological theory is used to describe its mechanical properties. The common constitutive models of silica gel materials are the Mooney Rivlin model, Ogden model, Yeoh model, etc.
[34-38]. Among them, the Yeoh model has good adaptability in the range of $300 \%$ deformation, which is the first choice for analyzing the deformation of silica gel.

Based on the stress-strain theory, the constitutive relation of silica gel material is established, and it is assumed that the silica gel material is isotropic and incompressible. Equation (1) of the strain energy function is as follows:

$$
\left\{\begin{array}{l}
W=W\left(I_{1}, I_{2}, I_{3}\right), \\
I_{1}=\lambda_{1}^{2}+\lambda_{2}^{2}+\lambda_{3}^{2}, \\
I_{2}=\lambda_{1}^{2} \lambda_{2}^{2}+\lambda_{2}^{2} \lambda_{3}^{2}+\lambda_{1}^{2} \lambda_{3}^{2}, \\
I_{3}=\lambda_{1}^{2} \lambda_{2}^{2} \lambda_{3}^{2},
\end{array}\right.
$$

where $I_{1}, I_{2}$, and $I_{3}$ are the invariants of the deformation tensor; $\lambda_{1}, \lambda_{2}$, and $\lambda_{3}$ are the main elongation ratio in three directions of space; and $W$ is the strain energy function.

According to the isotropy and incompressibility of the materials, Equation (2) is listed as follows:

$$
I_{3}=\lambda_{1}^{2} \lambda_{2}^{2} \lambda_{3}^{2}=1
$$

During the expansion of the driving cavity structure, the expansion wall will not only elongate but also will become thinner. So, it is necessary to assume that the expansion wall does not deform in the width direction, that is, $\lambda_{3}=1$. Therefore, according to the incompressibility of the material constitutive model, Equation (3) is listed as follows:

$$
\lambda_{2}^{2}=\frac{1}{\lambda_{1}^{2}} \text {. }
$$




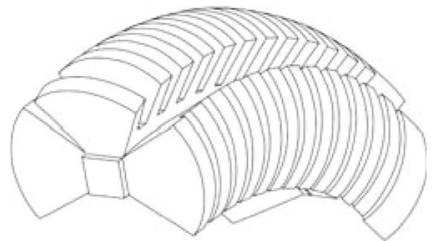

(a)

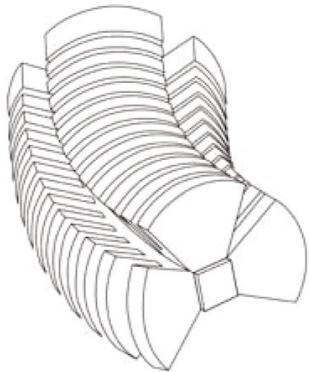

(b)

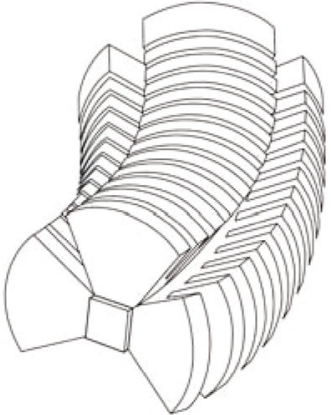

(c)

FIGURE 2: Periodic motion process diagram of pneumatic soft-bodied bionic actuator. (a) Middle part bending, (b) Left side bending. (c) Right side bending.

The deformation tensor can be expressed as in

$$
I_{1}=I_{2}=\lambda_{1}^{2}+\frac{1}{\lambda_{1}^{2}}+1
$$

Based on the most typical second-order parameters form of the Yeoh model, Equation (5) of the strain energy density function model is expressed as

$$
\begin{aligned}
W & =C_{10}\left(I_{1}-3\right)+C_{20}\left(I_{1}-3\right)^{2} \\
& =C_{10}\left(\lambda_{1}-\frac{1}{\lambda_{1}}\right)^{2}+C_{20}\left(\lambda_{1}-\frac{1}{\lambda_{1}}\right)^{4},
\end{aligned}
$$

where $C_{i j}$ is the dimensionless relationship constant, $C_{10}=$ $0.09, C_{20}=0.02$.

Combining Equation (1) to Equation (5), the expression of principal stress can be derived from the Piola-Kirchhoff stress and Cauchy-Green strain relationship, and thus, Equation (6) is listed as follows:

$$
\sigma_{i j}=\frac{2}{\lambda_{1}}\left(\lambda_{1}^{2}-\frac{1}{\lambda_{1}^{2} \lambda_{2}^{2}}\right)\left(\frac{\partial W}{\partial I_{1}}+\lambda_{2}^{2} \frac{\partial W}{\partial I_{2}}\right) .
$$

Substituting formula Equation (3) to Equation (5) into Equation (6), the relationship between stress and the main elongation ratio, i.e., Equation (7) is listed as follows:

$$
\sigma=\frac{\lambda_{1}^{4}-1}{\lambda_{1}^{3}}\left(2 C_{1}+4 C_{2}\left(\lambda_{1}-\frac{1}{\lambda_{1}}\right)^{2}\right) .
$$

3.2. Establishment of Mechanical Model. It is difficult to model the pneumatic soft-bodied bionic actuator, so it is necessary to simplify it properly in the nonlinear mechanical analysis. Since the actuator is composed of three identical actuating units arranged at equal angles, and driven in different time sequences according to the specific operation requirements, the equivalent bending deformation diagram can be obtained by taking the inflation of a single actuating unit as an example for theoretical modeling, as shown in Figure 3. Among them, the overall bending angle is indicated

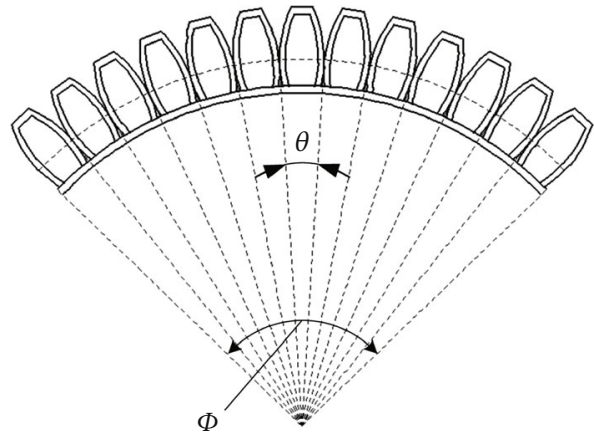

FIgURE 3: Expansion equivalent diagram.

by $\Phi$, and the bending angle of the single driving cavity structure is indicated by $\theta$.

According to Figure 3, the overall bending angle is the result of the bending superposition of each driving cavity structure, and the relationship between the overall bending angle $\Phi$ and $\theta$ can be expressed as in

$$
\Phi=13 \theta
$$

Then, the structure of a single driving cavity is researched, and the equivalent model of the initial state and expansion deformation state is obtained, as shown in Figure 4.

When the expansion wall expands outwards under the action of inflation pressure $P$, the lateral distance of expansion is $t_{a}$.

The bending angle $\theta$ of a single driving cavity structure can be expressed as in

$$
\theta=2 \arctan \left(\frac{t_{a}}{\left(\left(h-h_{a}\right) / 2\right)+h_{a}}\right) .
$$

Then, take the bottom end of the expansion wall of the driving cavity structure as the coordinate origin, and thus, the coordinate system is established, as shown in Figure 5. Among them, the center angle formed by the bulge of the expansion wall is $\delta$, the radius of the arc is $R$, the height of the expansion wall elongates from $h$ to the arc length $h_{b}$ after inflation, and the center coordinate is $\left(x_{0}, y_{0}\right)$. 


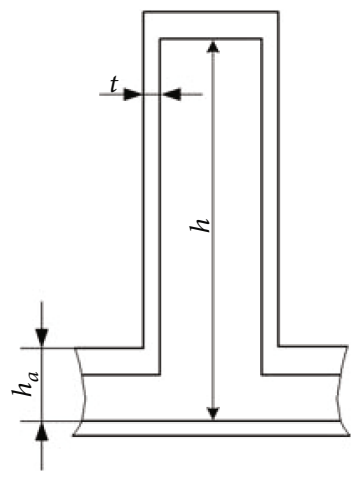

(a)

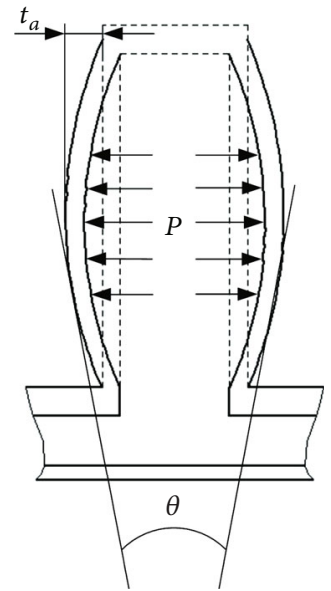

(b)

Figure 4: Equivalent diagram of a single driving cavity structure. (a) Initial state. (b) Deformation state.

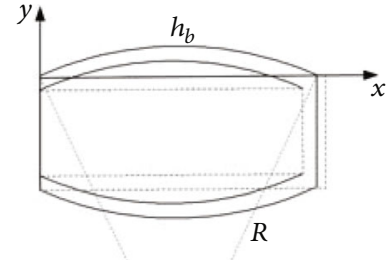

$\delta$

FIGURE 5: Coordinate system of a single driving cavity structure.

Then, the relation between $h_{b}$ and $h$ is expressed as in

$$
h_{\mathrm{b}}=\lambda_{1} h
$$

In the established coordinate system, the arc of the expansion wall can be expressed as in

$$
y=\sqrt{R^{2}-\left(x-x_{0}\right)^{2}}+y_{0},
$$

Then, according to the geometric relationship, Equation (12) is obtained as follows:

$$
\left\{\begin{array}{l}
x_{0}=\frac{h-h_{a}}{2}, \\
y_{0}=-R \cos \left(\frac{\delta}{2}\right), \\
R=\frac{h-h_{a}}{2 \cdot \sin (\delta / 2)} .
\end{array}\right.
$$

It can be expressed as in

$$
y\left(\frac{h-h_{a}}{2}\right)=\sqrt{R^{2}-\left(\frac{h-h_{a}}{2}-x_{0}\right)^{2}}+y_{0}=y(\delta) .
$$

Combining Equation (8) to Equation (13), the relationship between $\Phi$ and $\delta$ can be obtained as in

$$
\Phi=\Phi(\delta) .
$$

Through the relationship between the inflation pressure $P$ and the center angle $\delta$ of the expansion wall bulge, the relationship between the inflation pressure $P$ and the overall bending angle $\Phi$ is obtained. Then, assuming that the inflation pressure increases from 0 to $P$, and the deformation of each point on the expansion wall increases linearly to $y(x)$, the work done by the pressure at each point on the side wall to the side wall can be expressed as in

$$
U=26 \int_{0}^{h_{b}} P \cdot y(x) d x=26 P \int_{0}^{\lambda_{1} h}\left(\sqrt{R^{2}-\left(x-x_{0}\right)^{2}}+y_{0}\right) \mathrm{d} x=U(P, \delta) .
$$

The main elongation $\lambda_{1}$ is expressed by $\delta$, as in

$$
\lambda_{1}=\frac{\delta}{2 \cdot \sin (\delta / 2)} .
$$

Taking Equation (3) to Equation (5) into consideration, the function of strain energy density can be expressed as a function of $\delta$, as in

$$
W=W(\delta) .
$$

The deformation energy of the expansion wall after deformation can be expressed as in

$$
V=26 t \cdot\left(h-h_{a}\right) \cdot W(\delta)=V(\delta) .
$$

The deformation energy of the nonretractable column corresponding to the deformation of the expansion wall can be expressed as in

$$
E=l \cdot a \cdot W(\delta)=E(\delta) .
$$




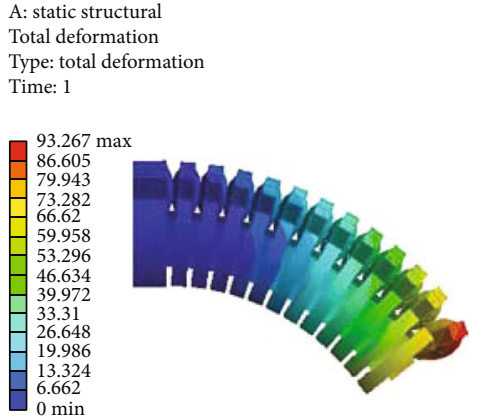

(a)

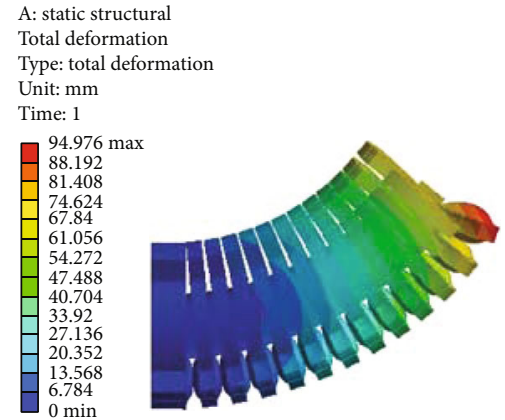

(b)

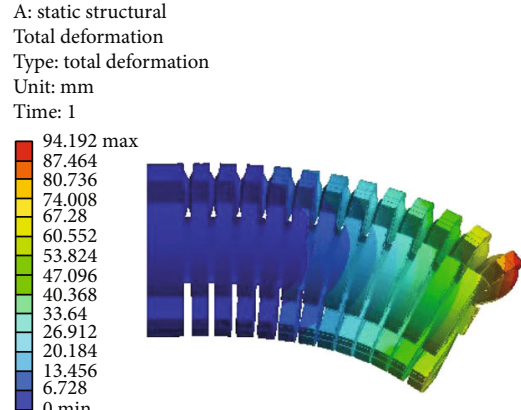

(c)

Figure 6: Pressurize expansion and bending of pneumatic soft-bodied bionic actuator. (a) Middle part actuating unit. (b) Left side actuating unit. (c) Right side actuating unit.

TABLE 2: Numerical simulation algorithm calculation results.

\begin{tabular}{lcccccccc}
\hline Driving pressure $(P, \mathrm{kPa})$ & 10 & 20 & 30 & 40 & 50 & 60 & 70 & 80 \\
Bending angle $(\Phi, \mathrm{rad})$ & 0.37 & 0.75 & 1.17 & 1.64 & 2.23 & 2.57 & 2.76 & 2.88 \\
\hline
\end{tabular}

The work done by the compression spring corresponding to the deformation of the expansion wall is

$$
E_{1}(\delta)=\frac{1}{2 a} k l^{2}
$$

where $k$ is the elasticity coefficient, the value is 1.1 .

According to the law of conservation of energy, the work done at each point on the expansion wall is equal to the deformation energy of the side wall of the driving cavity structure, and therefore, Equation (21) is listed as follows:

$$
V(\delta)=U(P, \delta)-E(\delta)-E_{1}(\delta)
$$

Combining Equation (14) and Equation (21) can further derive the function expression between the bending angle $\Phi$ and the inflation pressure $P$; this paper does not need to elaborate but uses Equation (22) to express it.

$$
\Phi=\Phi(P)
$$

\section{Numerical Simulation Algorithm Verification and Physical Model Preparation}

4.1. Verification of Numerical Simulation Algorithm. Based on Yeoh's second-order constitutive model of silica gel material for the pneumatic soft-bodied bionic actuator, the rationality of structural design and motion forms and the accuracy of the theoretical model of deformation analysis are verified by the numerical simulation algorithm [39-41]. The calculation results obtained from the numerical simulation algorithm are shown in Figure 6.

According to Figure 6, the deformation of the pneumatic soft-bodied bionic actuator conforms to the actual deformation of the silica gel material after inflation pressure and can meet the analysis results of motion forms. Therefore, the

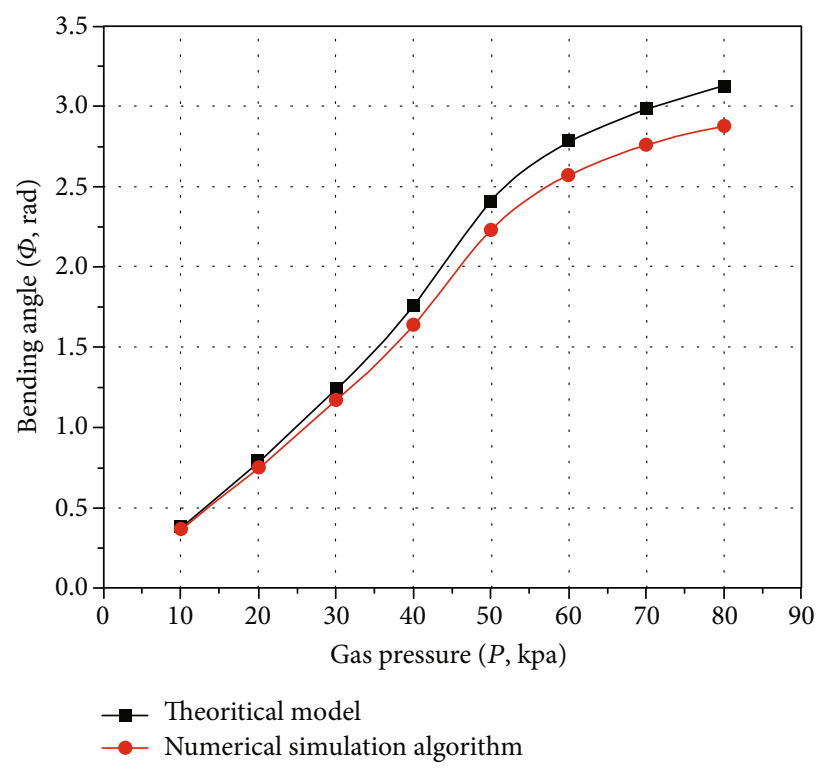

\begin{tabular}{|c|c|}
\hline Preparation objects & Material details \\
\hline Pneumatic network device & $\begin{array}{l}601 \text { room temperature-vulcanized } \\
\text { silica gel }\end{array}$ \\
\hline Nonretractable column & $\begin{array}{l}601 \text { room temperature-vulcanized } \\
\text { silica gel }\end{array}$ \\
\hline Compression spring & Quenched $65 \mathrm{Mn}$ \\
\hline Mould & PLA and paraffin wax \\
\hline
\end{tabular}

FIgURE 7: Error analysis of theoretical deformation analysis model.

TABle 3: Material preparation.

deformation change law of the cloud diagram can verify the rationality of the structure design and motion forms of the actuator. 


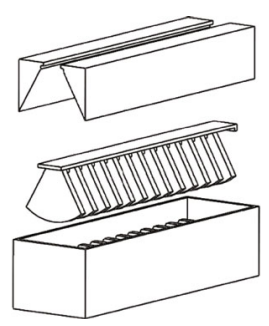

(a)

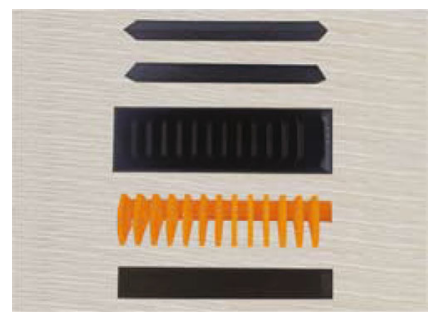

(b)

FIGURE 8: Mould structure. (a) Mould design of pneumatic network device. (b) Mould physical model.

It should be noted that the theoretical model of deformation analysis is based on the simplified equivalent bending deformation of the actuator, which is more similar to the deformation and bending of the middle part actuating unit of the actuator. Therefore, the theoretical model is verified by the numerical simulation results of deformation and bending of the middle part actuating unit of the actuator, and the numerical simulation algorithm calculation results under different driving pressures from $10 \mathrm{kPa}$ to $80 \mathrm{kPa}$ are obtained, as shown in Table 2.

By comparing the calculation results in Table 2 with the theoretical model of deformation analysis, as shown in Figure 7.

According to Figure 7, the calculation results of the numerical simulation algorithm and the theoretical model have the same curve change trend. Although the absolute error of the numerical simulation calculation results and the theoretical model gradually increases with the increase of the inflation pressure, the overall absolute errors change is still relatively accurate. The maximum absolute error is $0.24 \mathrm{rad}$, and the maximum relative error is $7.69 \%$. Therefore, the data measurement is relatively reliable and the theoretical model of deformation analysis has certain accuracy. Besides, it should be noted that the main reason for the errors is that the theoretical model only considers the expansion and bending changes of a single actuating unit.

4.2. Physical Model Preparation. The body structure of the pneumatic soft-bodied bionic actuator needs to have certain strength and flexibility. Deficiency in hardness results in overly soft structure, and consequently insufficient strength, and poor execution force, On the other hand, excessive hardness results in brittleness, making the structure easy to fracture [42-44]. Therefore, the room temperature-vulcanized silica gel with shore A20 hardness was selected.

4.2.1. Material Preparation. The materials required for the pneumatic soft-bodied bionic actuator are shown in Table 3 .

4.2.2. Mould Manufacture. According to the specific structural features of the actuator, based on 3D printing technology, the solid model of the mould is obtained [45-47], as shown in Figure 8. Among them, in order to demould smoothly, based on the lost wax casting technology, the mould core which forms the internal structure of the pneumatic network device is printed by paraffin wax material.

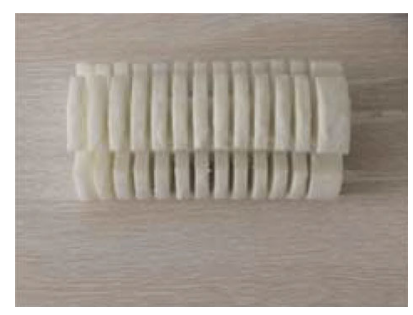

FIGURE 9: Physical model of pneumatic soft-bodied bionic actuator.

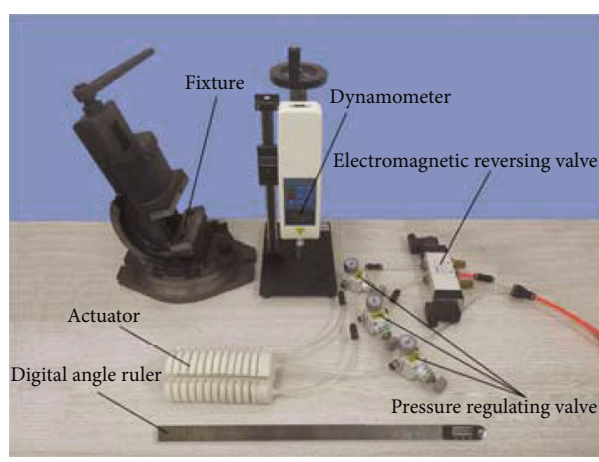

Figure 10: Experimental test platform.

4.2.3. Preparation of Physical Objects. Silica gel pouring, static forming, and demoulding are carried out based on the compound modeling process, and the core of the mould is melted by high-temperature melting technology, and the silica gel bonding technology is used for bonding [48]. Finally, the physical model of the pneumatic soft-bodied bionic actuator is completed, see Figure 9.

\section{Experimental Test and Analysis}

Through experimental testing and analysis, the exploration of the influence of different inflation pressure conditions on the motion and dynamic characteristics of the pneumatic softbodied bionic actuator lays the foundation for further application [49]. The main devices of the experiment include pneumatic soft-bodied bionic actuator, pressure regulating valves, electromagnetic reversing valve, fixture, digital angle ruler, and dynamometer. These devices are shown in Figure 10.

Among them, the inflatable end of the actuator is fixed and then the pressure from $10 \mathrm{kPa}$ to $80 \mathrm{kPa}$ is filled into the actuating unit by the air pressure pump.

\subsection{Kinematic Characteristics Analysis of Pneumatic Soft- Bodied Bionic Actuator}

5.1.1. Motion Frequency Analysis. On the premise of ensuring the same switching response time of the electromagnetic reversing valve, the motion frequency of the pneumatic soft-bodied bionic actuator is collected by the video camera. In the middle part periodic bending motion stage of actuator, the inflation pressure is decided, and deflation is conducted immediately after the completion of inflation; the experiment continues until the actuator reaches a straight state, so as to determine the whole cycle time, and to complete the research on the inflation and deflation of the middle part periodic 

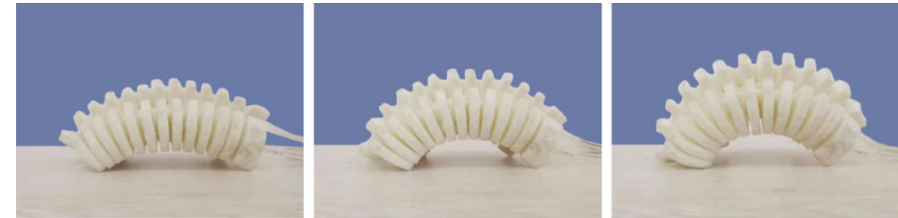

(a)

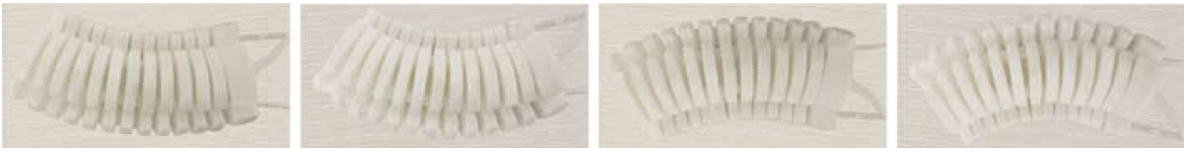

(b)

Figure 11: Motion process of pneumatic soft-bodied bionic actuator. (a) Middle part bending motion of actuator. (b) Left and right side bending motion of actuator.

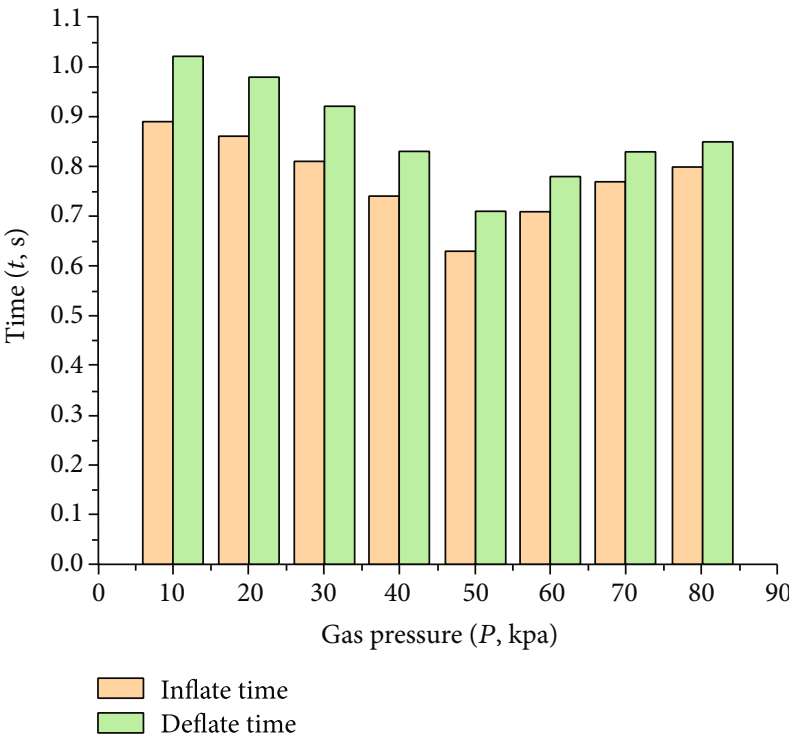

(a)

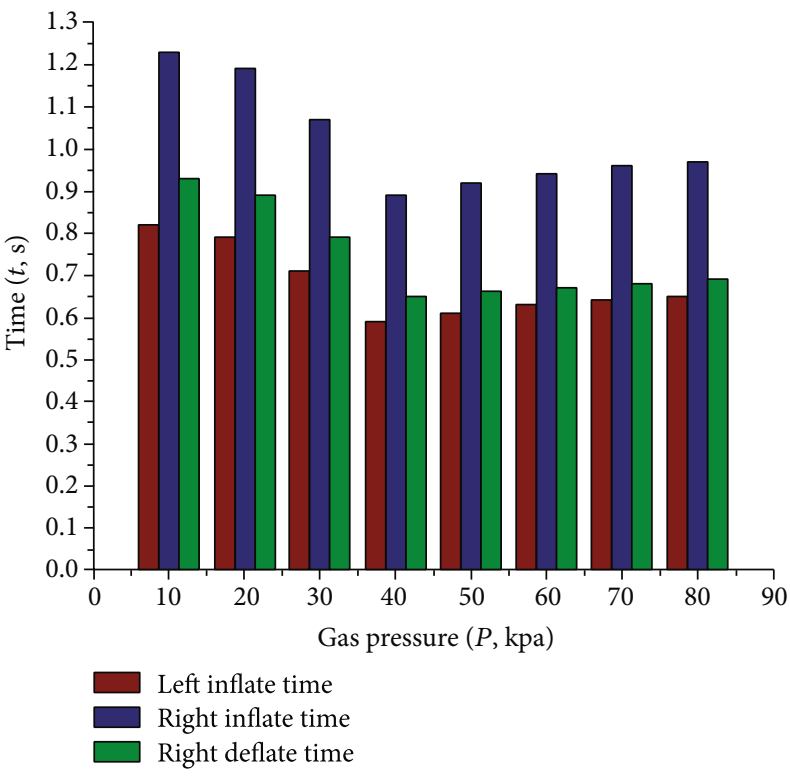

(b)

FIGURE 12: Motion cycle sequence diagrams of pneumatic soft-bodied bionic actuator. (a) Middle part bending motion cycle of actuator. (b) Left and right side bending motion cycle of actuator.

bending motion of the actuator, as shown in Figure 11(a). In the left and right side periodic bending motion stage of actuator, first the inflation pressure of actuator on one side is determined, and after inflation completes, it is deflated immediately, while the other side of actuator inflates. After inflation, the actuator deflates immediately until the end of deflation and the actuator reaches a straight state, as shown in Figure 11(b).

After data acquisition, the sequence diagram of the cycle of inflation and deflation of actuator is obtained, as shown in Figure 12.

According to Figure 12, the middle part periodic bending time of the pneumatic soft-bodied bionic actuator is shorter than that of the left and right sides, because it only does one-way bending motion. Besides, the unilateral motion time of the middle part periodic bending motion is longer than that of the left and right side periodic bending motion, which is caused by the larger amplitude of the middle part periodic bending motion compared with that of the left and right side periodic bending motion under the same inflation pressure. It should be noted that the periodic bending amplitude of the middle part increases as the inflation pressure increases, while the recovery force of the compression spring structure is also increasing, which makes the recovery time of the actuator structure gradually approximate the time of inflation bending gradually.

Then, according to the periodic sequence of actuator, the $P-f$ characteristic curves of inflation pressure can be obtained, as shown in Figure 13.

Figure 13 shows that the motion frequency of the pneumatic soft-bodied bionic actuator first increases and then decreases with the increase of inflation pressure, which is caused by the increase of its motion amplitude. When the motion amplitude is relatively small, the motion frequency increases with the increase of inflation pressure, and when the motion amplitude is relatively large, the motion 


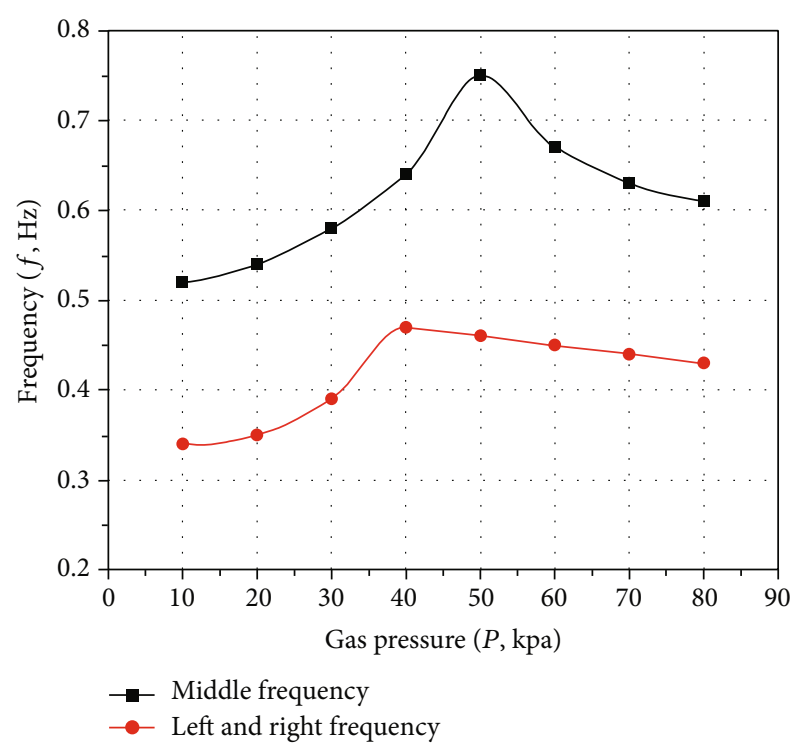

Figure 13: $P-f$ characteristic curves of pneumatic soft-bodied bionic actuator.

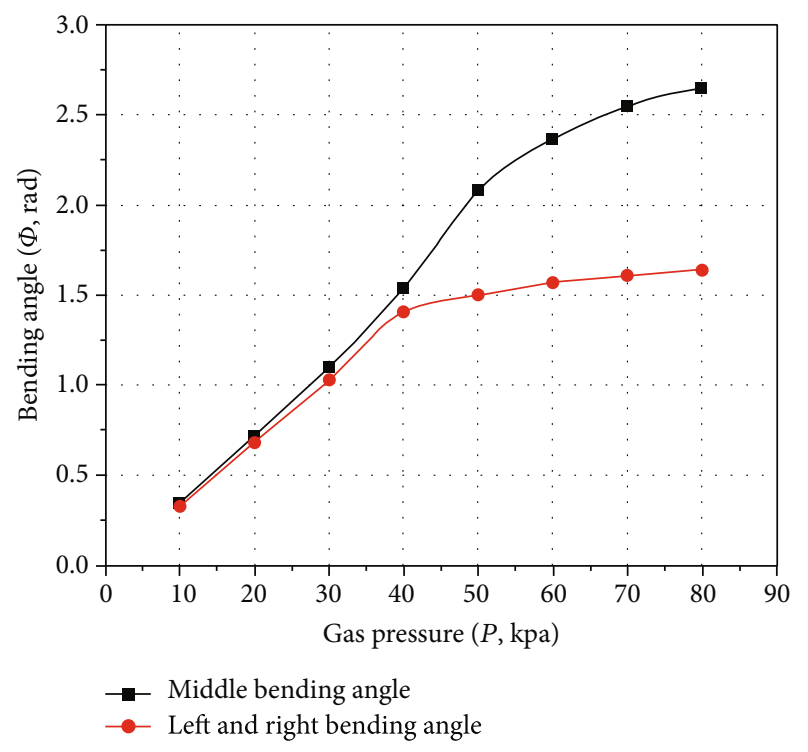

FIgURe 14: $P-\Phi$ characteristic curves of pneumatic soft-bodied bionic actuator.

frequency decreases with the increase of inflation pressure. Among them, the motion frequency of the middle part periodic bending is higher than that of the left and right side periodic bending.

5.1.2. Bending Angle Analysis. The $P-\Phi$ characteristic curves between the inflation pressure and the bending angles of the actuator can be obtained by collecting the bending angles of the pneumatic soft-bodied bionic actuator with the digital angle ruler, as shown in Figure 14.

Figure 14 shows that the maximum bending angles produced by the periodic bending motion on the middle part of the pneumatic soft-bodied bionic actuator and the periodic bending motion on the left and right sides are $2.65 \mathrm{rad}$ and $1.64 \mathrm{rad}$, respectively. Within this range, the periodic
TABLE 4: Empirical formula coefficients of $P-\Phi$ characteristic curves.

\begin{tabular}{lcc}
\hline $\begin{array}{l}\text { Empirical formula } \\
\text { coefficients }\end{array}$ & $\begin{array}{c}\text { Middle part } \\
\text { bending angle }\end{array}$ & $\begin{array}{c}\text { Left and right side } \\
\text { bending angle }\end{array}$ \\
\hline$p_{1}$ & $6.25 E-8$ & $2.28 E-7$ \\
$p_{2}$ & $-2.02 E-5$ & $-3.99 E-5$ \\
$p_{3}$ & $1.64 E-3$ & $1.95 E-3$ \\
$p_{4}$ & $-3.94 E-3$ & $1.27 E-3$ \\
$p_{5}$ & $2.57 E-1$ & $1.58 E-1$ \\
\hline
\end{tabular}

bending motion of middle part and the periodic bending motion of left and right sides can, respectively, guarantee the operation in the best state. Among them, the $P-\Phi$ characteristic curves change gradually from the initially linear to later nonlinear, due to the expansion of the actuating unit reaching a certain extent, and the large reverse bending motion produced by the nonretractable column of silica gel body and the compression spring structure. The bending angle of the left and right side periodic bending motion is smaller than that of the middle part periodic bending motion, because it also bears the reverse bending motion produced by the compressed side when bending. It should be noted that if the inflatable pressure continues to increase, although the bending angle of the actuator will continue to increase slowly, its motion characteristics will become worse, the execution ability will be weakened, and the operation effect will be affected to a certain extent.

According to the $P-\Phi$ characteristic curves, an empirical equation (23) can be obtained, which can reflect the change of its characteristics.

$$
f(x)=p_{1} \cdot x^{4}+p_{2} \cdot x^{3}+p_{3} \cdot x^{2}+p_{4} \cdot x+p_{5} .
$$

The specific scaling coefficients are shown in Table 4 .

The $P-\Phi$ characteristic curves obtained from the experimental test and the calculation results of the numerical simulation algorithm undergo the accuracy analysis of errors, and the results are shown in Figure 15.

According to Figure 15, the calculation results of the experimental test and the numerical simulation calculation results have the same curve change trend. Although the absolute errors of the experimental test and the numerical simulation calculation result gradually increase with the increase of the inflation pressure, the overall absolute errors change is still relatively accurate. The maximum absolute error is $0.23 \mathrm{rad}$, and the maximum relative error is $7.99 \%$. Therefore, the data measurement is relatively reliable, and the numerical simulation calculation results and the theoretical model of deformation analysis have certain accuracy. Besides, it should be noted that the main causes of experimental test errors are measurement errors, manufacturing errors, experimental instrument errors, etc.

5.2. Analysis of Dynamic Characteristics of Pneumatic SoftBodied Bionic Actuator. When analyzing the dynamic characteristics of pneumatic soft-bodied bionic actuator, the 


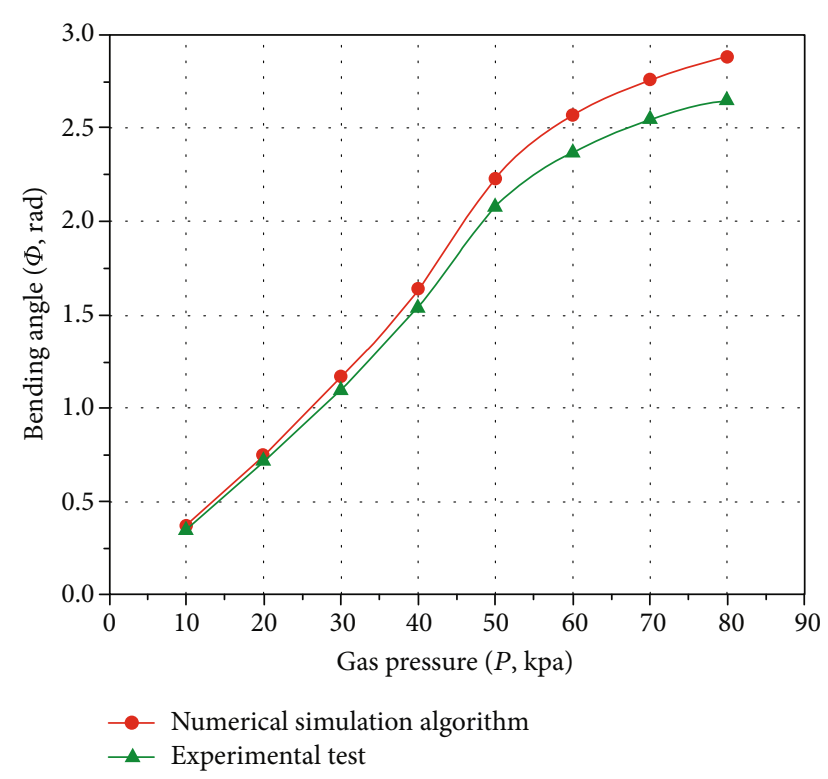

FIGURE 15: Errors analysis of numerical simulation deformation model.

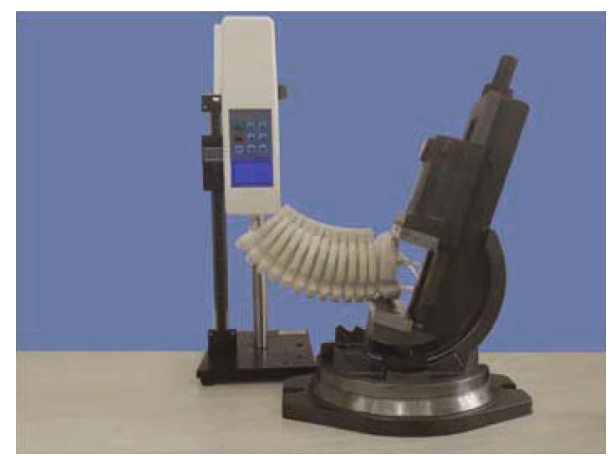

Figure 16: Driving torque measure experiment of pneumatic softbodied bionic actuator.

driving torque is collected by dynamometer, as shown in Figure 16.

5.2.1. Dynamic Characteristic Analysis of Variable Bending Angle. The bending angle of the actuator is variable; the $P$ $-M$ characteristic curves of inflation pressure and driving torque are obtained, as shown in Figure 17.

Figure 17 shows that the driving torque values of the left and right side periodic bending of the pneumatic soft-bodied bionic actuator is relatively lower compared with that of the middle part periodic bending, which is caused by the counter torque produced by the compressed side cavity structure. It should be noted that the variation trend of the $P-M$ characteristic curves is similar to that of the $P-\Phi$ characteristic curves, which indicates that the driving torque is closely related to the bending angle. In addition, with the increase of inflation pressure, the nonlinear change is partly due to the extreme deformation of the actuator body structure, which leads to the change of its stress form.

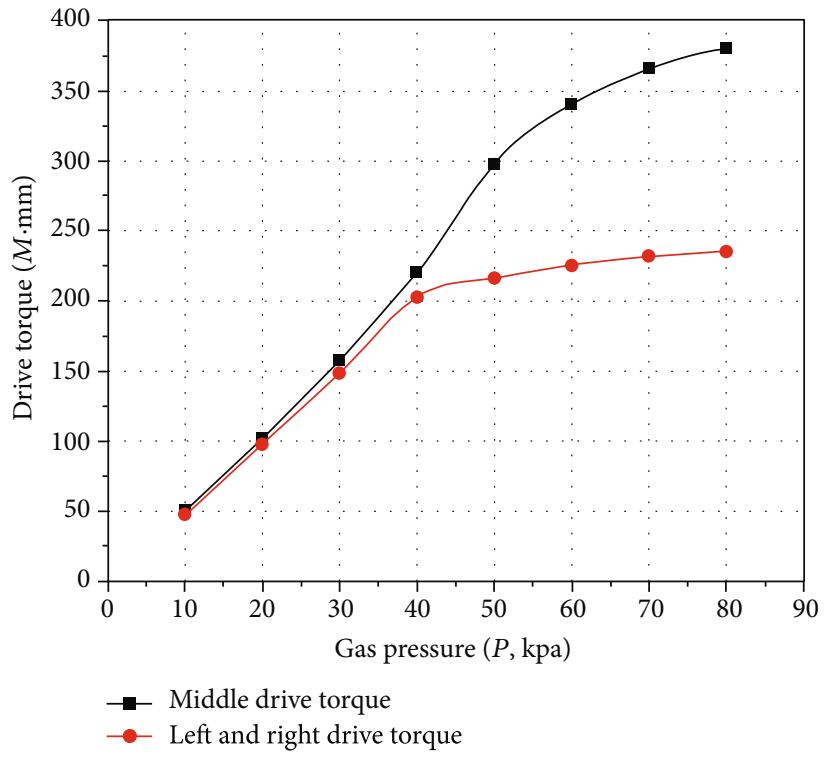

Figure 17: $P-M$ characteristic curves of pneumatic soft-bodied bionic actuator for variable angle.

TABle 5: Empirical formula coefficients of $P-M$ characteristic curves.

\begin{tabular}{lcc}
\hline $\begin{array}{l}\text { Empirical formula } \\
\text { coefficients }\end{array}$ & $\begin{array}{c}\text { Middle part } \\
\text { bending angle }\end{array}$ & $\begin{array}{c}\text { Left and right side } \\
\text { bending angle }\end{array}$ \\
\hline$p_{1}$ & $9.36 E-6$ & $3.26 E-5$ \\
$p_{2}$ & $-2.99 E-3$ & $-5.72 E-3$ \\
$p_{3}$ & $2.43 E-1$ & $2.79 E-1$ \\
$p_{4}$ & $-7.80 E-1$ & $-2.02 E-1$ \\
$p_{5}$ & 38.29 & 22.7 \\
\hline
\end{tabular}

The $P-M$ characteristic curves are expressed by the empirical equation (23), and the specific scale coefficients are shown in Table 5.

5.2.2. Dynamic Characteristic Analysis of Fixed Bending Angle. The bending angle of the actuator is fixed; the $P-M$ characteristic curves of inflation pressure and driving torque are obtained, as shown in Figure 18.

According to Figure 18, the middle part driving torques of the actuator and the left and right sides both increase linearly with the increase of the inflation pressure. It should be noted that the middle part driving torque of the actuator is larger than that of the left and right sides, because the left and right sides need to bear the reverse bending motion produced by the compressed side when bending; this phenomenon can correspond to the above test results.

\section{Conclusions}

This paper designs a kind of pneumatic soft-bodied bionic actuator, which is suitable for robot carriers of underwater swimming and land crawling. It has the advantages of simple 


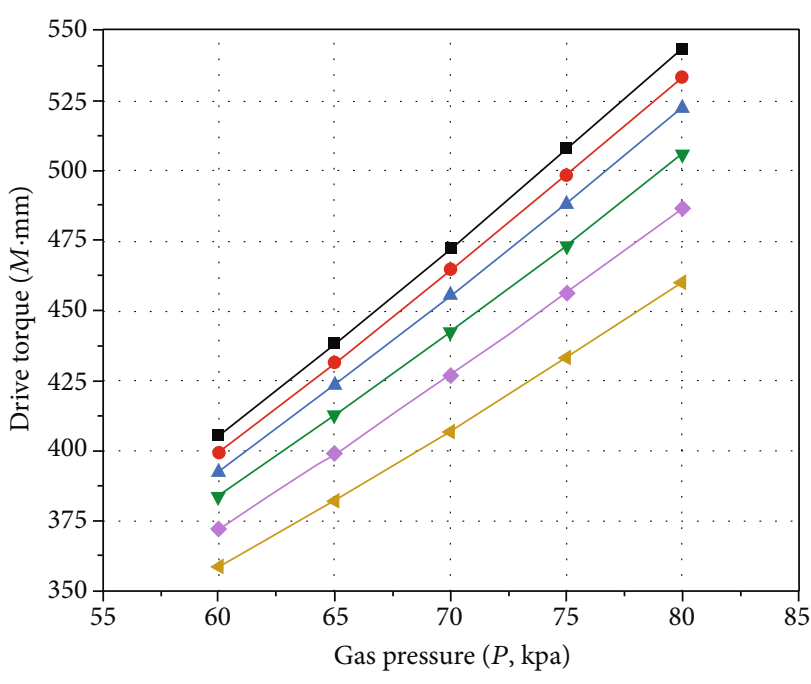

(a)

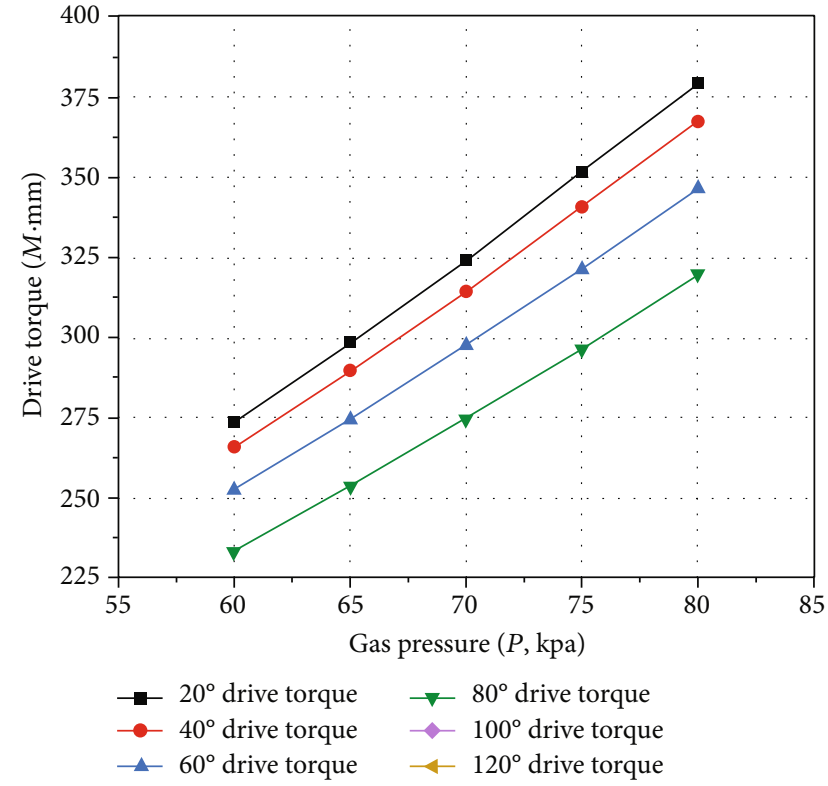

(b)

Figure 18: The $P-M$ characteristic curves of pneumatic soft-bodied bionic actuator for fixed angle. (a) Middle part driving torque. (b) Left and right side driving torque.

structure, strong environmental adaptability, high driving efficiency, etc.

(1) Based on Yeoh's second-order constitutive model of the silica gel material, the deformation analysis model of the actuator is established, and the relationship between the bending angle of the actuator and the inflation pressure is determined, which can provide a theoretical reference for the structural design and deformation analysis of the actuator of the softbodied bionic robot

(2) Using the numerical simulation algorithm, the rationality of the actuator structure design and motion form is verified, and the accuracy of the theoretical model of deformation analysis is determined, and the accuracy of the deformation analysis theoretical model is determined through the error accuracy analysis results of the numerical simulation algorithm and experimental test. Among them, the maximum absolute error and relative error between the calculation results of the numerical simulation algorithm and theoretical model are 0.24 radian and $7.69 \%$, and the maximum absolute error and relative error of the experimental test and calculation results of numerical simulation algorithm are 0.23 radian and $7.99 \%$

(3) The physical model of the actuator is verified and analyzed via the experimental test platform. According to the values of inflation pressure filled into the actuating unit, the motion and dynamic output in a certain range are matched, and the characteristic curves of the motion frequency, bending angle and driving torque of the actuator, and the correlative empirical formulas are obtained. The accuracy of the deformation analysis model and the numerical simulation algorithm is verified, and the actuator proves to be reasonable and feasible, which can be effectively used as the actuator of the soft-bodied bionic robot

\section{Data Availability}

All data generated or analyzed used to support the findings of this study are included within the article.

\section{Conflicts of Interest}

The authors declare that there is no conflict of interest regarding the publication of this paper.

\section{Acknowledgments}

This work was supported by the Institute of Robotics in Shenyang University of Technology. This work was supported by the National Natural Science Foundation of China (general program) (grant number 51775354).

\section{References}

[1] B. Mosadegh, P. Polygerinos, C. Keplinger et al., "Soft Robotics: pneumatic networks for soft robotics that actuate rapidly (Adv. Funct. Mater. 15/2014)," Advanced Functional Materials, vol. 24, no. 15, pp. 2109-2119, 2014.

[2] C. Pengzhan, Z. Wuwei, Z. Jinglong, and Q. Fengyu, "Design and control of soft pneumatic fiber enforcement actuator," Journal of Nanjing University of Science and Technology, vol. 2, no. 40, pp. 218-222, 2016.

[3] L. Chen, C. Yang, H. Wang, D. T. Branson, J. S. Dai, and R. Kang, "Design and modeling of a soft robotic surface with 
hyperelastic material," Mechanism and Machine Theory, vol. 130, no. 13, pp. 109-122, 2018.

[4] A. D. Marchese, R. K. Katzschmann, and D. Rus, "A recipe for soft fluidic elastomer robots," Soft Robotics, vol. 2, no. 1, pp. 7$25,2015$.

[5] S. I. Rich, R. J. Wood, and C. Majidi, "Untethered soft robotics," Nature Electronics, vol. 1, no. 2, pp. 102-112, 2018.

[6] S. W. Yeom and I. K. Oh, "A biomimetic jellyfish robot based on ionic polymer metal composite actuators," Smart Material Structures, vol. 18, no. 8, pp. 085002-085092, 2009.

[7] J. Najem and D. J. Leo, A bio-inspired bell kinematics design of a jellyfish robot using ionic polymer metal composites actuators, Proc SPIE, 2012.

[8] R. F. Shepherd, A. A. Stokes, J. Freake et al., "Using explosions to power a soft robot," Angewandte Chemie International Edition, vol. 52, no. 10, pp. 2892-2896, 2013.

[9] A. A. Villanueva, K. J. Marut, T. Michael, and S. Priya, "Biomimetic autonomous robot inspired by theCyanea capillata(Cyro)," Bioinspiration \& Biomimetics, vol. 8, no. 4, pp. 046005-046055, 2013.

[10] F. Renda, M. Giorelli, M. Calisti, M. Cianchetti, and C. Laschi, "Dynamic model of a multibending soft robot arm driven by cables," IEEE Transactions on Robotics, vol. 30, no. 5, pp. 1109-1122, 2014.

[11] Y. Bahramzadeh and M. Shahinpoor, "A review of ionic polymeric soft actuators and sensors," Soft Robotics, vol. 1, no. 1, pp. 38-52, 2014.

[12] M. Wehner, R. L. Truby, D. J. Fitzgerald et al., "An integrated design and fabrication strategy for entirely soft, autonomous robots," Nature, vol. 536, no. 7617, pp. 451-455, 2016.

[13] H. Rodrigue, W. Wang, M. W. Han, Y. J. Quan, and S. H. Ahn, "Comparison of mold designs for SMA-based twisting soft actuator," Sensors and Actuators A: Physical, vol. 237, no. 7, pp. 96-106, 2016.

[14] H. Wang, Y. Yao, X. Wang et al., "Large-magnitude transformable liquid-metal composites," ACS Omega, vol. 4, no. 1, pp. 2311-2319, 2019.

[15] I. Must, E. Sinibaldi, and B. Mazzolai, "A variable-stiffness tendril-like soft robot based on reversible osmotic actuation," Nature Communications, vol. 10, no. 1, pp. 1-9, 2019.

[16] Y. Wu, J. K. Yim, J. Liang et al., "Insect-scale fast moving and ultrarobust soft robot," Science robotics, vol. 4, no. 32, pp. eaax1594-eaax1611, 2019.

[17] A. D. Marchese, C. D. Onal, and D. Rus, "Autonomous soft robotic fish capable of escape maneuvers using fluidic elastomer actuators," Soft Robotics, vol. 1, no. 1, pp. 75-87, 2014.

[18] A. Jusufi, D. M. Vogt, R. J. Wood, and G. V. Lauder, "Undulatory swimming performance and body stiffness modulation in a soft robotic fish-inspired physical model," Soft Robotics, vol. 4, no. 3, pp. 202-210, 2017.

[19] J. Frame, N. Lopez, O. Curet, and E. D. Engeberg, "Thrust force characterization of free-swimming soft robotic jellyfish," Bioinspiration \& Biomimetics, vol. 13, no. 6, pp. 26-44, 2018.

[20] M. T. Tolley, R. F. Shepherd, B. Mosadegh et al., "A resilient, untethered soft robot," Soft Robotics, vol. 1, no. 3, pp. 213223, 2014.

[21] S. Yanqiong, P. Wu, and Y. Wenbo, "Movement of air-driven soft robot," Journal of Mechanical Engineering, vol. 53, no. 13, pp. 14-18, 2017.

[22] S. Liming, L. Tingyu, and X. I. Zuoyan, "Analysis and experiment of actuating method for pneumatic soft crawling robot,"
Chinese Hydraulic \& Pneumatics, vol. 1, no. 11, pp. 99-103, 2018.

[23] M. J. Bishop and S. Kota, "Towards snake-like soft robots: design of fluidic fiber-reinforced elastomeric helical manipulators," in 2013 IEEE/RSJ International Conference on Intelligent Robots and Systems, Tokyo, Japan, 2013.

[24] M. Luo, W. Tao, F. Chen, T. K. Khuu, S. Ozel, and C. D. Onal, "Design improvements and dynamic characterization on fluidic elastomer actuators for a soft robotic snake," in 2014 IEEE International Conference on Technologies for Practical Robot Applications (TePRA), Woburn, MA, USA, 2014.

[25] Y. Jiantao, C. Xinbo, C. Juntao, Z. Hong, L. Haili, and Z. Yongsheng, "Design and motion analysis of a wheelwalking bionic soft robot," Journal of Mechanical Engineering, vol. 55, no. 5, pp. 27-35, 2019.

[26] F. Jizhuang, Y. Qingguo, Y. Bowen, and Z. Jie, “Development of a joint-like pneumatic actuator applied to soft frog-inspired swimming robot," Robot, vol. 40, no. 5, pp. 4-12, 2018.

[27] J. B. Moser, G. Krishnan, C. Kim, and S. Kota, "Design of soft robotic actuators using fluid-filled fiber-reinforced elastomeric enclosures in parallel combinations," in IEEE/RSJ International Conference on Intelligent Robots and Systems, Vilamoura, Portugal, 2012.

[28] A. D. Marchese, R. K. Katzschmann, and D. L. Rus, "Whole arm planning for a soft and highly compliant 2D robotic manipulator," in 2014 IEEE/RSJ International Conference on Intelligent Robots and Systems, Chicago, IL, USA, 2014.

[29] Y. Hao, Z. Gong, Z. Xie et al., "Universal soft pneumatic robotic gripper with variable effective length," in 2016 35th Chinese Control Conference (CCC), Chengdu, China, 2016.

[30] Z. Wang and S. Hirai, "Soft gripper dynamics using a linesegment model with an optimization-based parameter identification method," IEEE Robotics and Automation Letters, vol. 2, no. 2, pp. 624-631, 2017.

[31] B. N. Peele, T. J. Wallin, H. Zhao, and R. F. Shepherd, "3D printing antagonistic systems of artificial muscle using projection stereolithography," Bioinspiration \& Biomimetics, vol. 10, no. 5, pp. 45-53, 2015.

[32] S. Liming, X. Zuoyan, and L. Tingyu, "Design and fabrication of multi-chamber biomimetic pneumatic soft actuators," Chinese Journal of Engineering Design, vol. 1, no. 5, pp. 31-37, 2017.

[33] B. T. Phillips, K. P. Becker, S. Kurumaya et al., "A dexterous, glove-based teleoperable low-power soft robotic arm for delicate deep-sea biological exploration," Scientific Reports, vol. 8, no. 1, article 14779, 2018.

[34] O. D. Yirmibesoglu, J. Morrow, S. Walker et al., "Direct 3D printing of silicone elastomer soft robots and their performance comparison with molded counterparts," in 2018 IEEE International Conference on Soft Robotics (RoboSoft), Livorno, Italy, 2018.

[35] Y. Mei, B. Stover, N. Afsar Kazerooni et al., "A comparative study of two constitutive models within an inverse approach to determine the spatial stiffness distribution in soft materials," International Journal of Mechanical Sciences, vol. 140, pp. 446454, 2018.

[36] S. K. Panda and M. L. Buist, "A finite nonlinear hyperviscoelastic model for soft biological tissues," Journal of Biomechanics, vol. 69, pp. 121-128, 2018.

[37] K. Matouš, M. G. D. Geers, V. G. Kouznetsova, and A. Gillman, "A review of predictive nonlinear theories for 
multiscale modeling of heterogeneous materials," Journal of Computational Physics, vol. 330, no. 5, pp. 192-220, 2017.

[38] A. Chanda and C. Callaway, "Tissue anisotropy modeling using soft composite materials," Applied Bionics and Biomechanics, vol. 2018, no. 18, pp. 1-9, 2018.

[39] Y. T. Kao, Y. Zhang, J. Wang, and B. L. Tai, "Bending behaviors of 3D-printed bi-material structure: experimental study and finite element analysis," Additive Manufacturing, vol. 16, pp. 197-205, 2017.

[40] W. M. Chen, T. Lee, V. S. Lee, J. W. Lee, and S. J. Lee, "Effects of internal stress concentrations in plantar soft-tissue-a preliminary three-dimensional finite element analysis," Medical Engineering \& Physics, vol. 32, no. 4, pp. 324-331, 2010.

[41] S. Kindo, R. Vashistha, S. Raj, G. P. Ravishankar, and M. P. Padwale, "Selection and validation of hyperelastic finite element model for analysis of silicone rubber," in AIP Conference Proceedings, Thessaloniki, Greecereference, 2018.

[42] Q. Xu, J. Liu, and L. Qu, "Dynamic modeling for silicone beams using higher-order ANCF beam elements and experiment investigation," Multibody System Dynamics, vol. 46, no. 4, pp. 307-328, 2019.

[43] R. K. Katzschmann, A. D. Maille, D. L. Dorhout, and D. Rus, "Cyclic hydraulic actuation for soft robotic devices," in 2016 IEEE/RSJ International Conference on Intelligent Robots and Systems (IROS), Daejeon, South Kore, 2016.

[44] W. Wang, J. Y. Lee, H. Rodrigue, S. H. Song, W. S. Chu, and S. H. Ahn, "Locomotion of inchworm-inspired robot made of smart soft composite (SSC)," Bioinspiration \& Biomimetics, vol. 9, no. 4, pp. 46-66, 2014.

[45] M. Launhardt, N. Ebel, M. Kondruweit, M. Weyand, T. Volk, and D. Drummer, "Developing a patient individualized flexible silicone implant using SLS and vacuum die casting," in AIP Conference Proceedings, Dresden, Federal Republic of Germany, 2019.

[46] B. S. Homberg, R. K. Katzschmann, M. R. Dogar, and D. Rus, "Haptic identification of objects using a modular soft robotic gripper," in 2015 IEEE/RSJ International Conference on Intelligent Robots and Systems (IROS), Hamburg, Germany, 2015.

[47] R. K. Katzschmann, A. D. Marchese, and D. Rus, "Hydraulic autonomous soft robotic fish for 3D swimming," in Experimental Robotics, pp. 405-420, Springer, Cham, 2016.

[48] Y. Staudt, C. Odenbreit, and J. Schneider, "Failure behaviour of silicone adhesive in bonded connections with simple geometry," International Journal of Adhesion and Adhesives, vol. 82, pp. 126-138, 2018.

[49] G. Agarwal, N. Besuchet, B. Audergon, and J. Paik, "Stretchable materials for robust soft actuators towards assistive wearable devices," Scientific Reports, vol. 6, no. 1, pp. 324-341, 2016. 